\title{
Isolation and characterization of tomato cDNA and genomic clones encoding the ubiquitin gene $u b i 3$
}

\author{
Neil E. Hoffman, ${ }^{1}$ Kenton Ko, ${ }^{2}$ Deborah Milkowski ${ }^{1}$ and Eran Pichersky ${ }^{3}$ \\ ${ }^{1}$ Department of Plant Biology, Carnegie Institute of Washington, 290 Panama Street, Stanford, CA \\ 94305, USA; ${ }^{2}$ Department of Biology, Queens University, Kingston, Ontario K7L 3N6, Canada; ${ }^{3}$ Biology \\ Department, University of Michigan, Ann Arbor, MI 48109, USA
}

Received 5 March 1991; accepted in revised form 22 July 1991

Key words: ribosomes, chloroplast, expression library, $r b c S$, GT-1, AT-1

\begin{abstract}
We report here the isolation and nucleotide sequence of tomato cDNA and genomic clones encoding a ubiquitin extension protein homologous to the yeast gene $u b i 3$. Sites similar to upstream activating sites commonly found in the promoters of yeast ribosomal genes were observed in the tomato promoter. The tomato $u b i 3$ promoter also contained elements found in the $r b c S$ promoter from pea. The transcription initiation site was determined to occur 66 bp upstream of the initiating Met. RFLP mapping revealed that the gene was located on chromosome 1,23 cM from marker TG301. A ubi3 gene-specific probe hybridized to a single $800 \mathrm{nt}$ transcript. Expression was reduced in heat-shocked plants and plants kept in the dark. Expression was highest in young leaves and immature green fruit and lowest in mature leaves and petals. We isolated the original cDNA clone using an antibody prepared against chloroplast polypeptides. Immunological studies did not detect ubiquitin or ubiquitin extension proteins in the chloroplast. However, higher-molecular-weight chloroplast proteins were detected with ubiquitin antisera suggesting that ubiquitin conjugates are transported into the chloroplast.
\end{abstract}

\section{Introduction}

Ubiquitin is a highly conserved 76-residue protein found in all eukaryotic cells. It is found free or covalently joined, through its carboxyl terminal glycine residue, to the $\varepsilon$-amino group of a lysine residue in various cytoplasmic, nuclear, and in- tegral membrane proteins [6]. Proteins conjugated to multiubiquitin chains are selectively degraded while proteins conjugated to monoubiquitin are often stabilized [6].

In yeast, four ubiquitin genes have been isolated [26] all of which encode ubiquitin polyproteins. The yeast $u b i 4$ gene encodes 5 copies of

The nucleotide sequence data reported will appear in the EMBL, GenBank and DDBJ Nucleotide Sequence Databases under the accession number X58253. 
ubiquitin tandemly linked whereas $u b i l, u b i 2$, and $u b i 3$ encode polyproteins consisting of a single copy of ubiquitin fused to highly basic, cysteinerich polypeptides. The yeast ubil and ubi2 genes encode ubiquitin fused to a 52 amino acid $\mathrm{C}$ terminal extension, whereas $u b i 3$ encodes a 76 amino acid extension with little sequence similarity to the extensions of the UBI1 and UBI2 proteins. Recently it was demonstrated that the 52 and 76 amino acid extensions of UBI1, UBI2, and UBI3 are polypeptide components of the $80 \mathrm{~S}$ ribosomes $[9,23,30]$. Furthermore, the incorporation of the extensions into ribosomes was promoted by their association with ubiquitin, leading Finley et al. [9] to suggest a novel chaperone function for ubiquitin. Ubiquitin has also been found to promote the translocation of monoamine oxidase $B$ into the mitochondrial outer membrane [37], a function characteristic of other chaperone-like proteins $[7,25,29]$.

In plants, ubiquitin is known to play a role in the degradation of the Pfr form of phytochrome [32]. Plant ubiquitin genes homologous to ubil and $u b i 2$ [3, 13], ubi3 [4, 12] and ubi4 [2] have recently been cloned and sequenced. Callis et al. [4] have also provided evidence that the extension proteins are associated with cytoplasmic ribosomes.

In this report we describe the cloning of a ubiquitin gene highly homologous to yeast $u b i 3$ that we isolated while screening a tomato cDNA library with antibodies made against chloroplast polypeptides from photosystem I. Unlike the yeast gene, the cDNA clone we isolated contained no stop codons upstream of the first methionine suggesting the possibility that plants contain a form of UBI3 that has a transit peptide and is targeted to the chloroplast. The recent report that Chlamydomonas chloroplasts contain protein that binds to ubiquitin antibody is also consistent with this notion [36]. To examine this possibility we further isolated and characterized a tomato ubi3 genomic clone. We present the gene sequence, its chromosomal location, transcription initiation site, and data on its expression during development and under stress. Our results indicate that the UBI3 protein does not contain a transit pep- tide. Furthermore, using ubiquitin antibodies as probes, we were unable to detect any free ubiquitin in the chloroplasts of spinach or pea. These antibodies, however, recognized several highermolecular-weight chloroplast proteins in both pea and spinach.

\section{Materials and methods}

\section{Library screening}

The construction of the tomato cDNA expression library and procedures employed for screening the library are described and referenced in Hoffman et al. [17]. The polyclonal antiserum used in the identification of cDNA clones was prepared against Vicia faba $14-20 \mathrm{kDa}$ photosystem I polypeptides. Briefly, photosystem I polypeptides were prepared according to Mullet et al. [24], electrophoresed on preparative denaturing SDSPAGE gels, and polypeptides in the $14-20 \mathrm{kDa}$ range were electroeluted from the gel and used as antigen in New Zealand rabbits. The tomato genomic library was a gift from Dr. W. Gruissem, Dept of Botany, University of California at Berkeley, CA [34]. The genomic library was plated using the Escherichia coli host Q358 and duplicate filters were screened using a 574 bp Bgl II-Sty I subfragment or a 282 bp Alu I-Sty I subfragment of the $u b i 3$ cDNA insert as radiolabelled probes as outlined in Maniatis et al. [22]. The Bgl IISty I probe hybridizes to all ubiquitin genes while the Alu I-Sty I probe is specific for ubi3. Filters were prehybridized for 10 min in $6 \times \mathrm{SSC}$ containing $0.25 \%$ non-fat milk and hybridized in the same buffer containing $10^{6} \mathrm{cpm} / \mathrm{ml}$ probe at $68^{\circ} \mathrm{C}$ for the large probe and $50^{\circ} \mathrm{C}$ for the small probe. Filters were washed in $0.1 \times$ SSC containing $0.1 \% \mathrm{SDS}$ at room temperature. Colonies detected on duplicate filters were plaque-purified and subcloned for further analysis.

The nucleotide sequence of the $u b i 3 \mathrm{cDNA}$ and genomic clones were determined by the dideoxynucleotide chain termination method on doublestranded DNA templates [31]. 


\section{Expression studies and primer extension}

Total plant RNA samples used for analyzing tissue-specific expression were isolated from field-grown tomato (Lycoperiscon esculentum cv. Big Girl; Burpee Seed Co). Leaf tissue was from either the youngest (first leaf) or fourth leaf from the meristem. Petioles were from the fourth leaf. Only fully open flowers were harvested. One collection comprised yellow flower petals only and the other contained a mixture of petals and ovaries. Fruit were designated stage 1 when green with immature seeds, stage 2 when green but seeds were fully developed, stage 3 when color was first evident, and stage 4 when fully red. Tissue was harvested between 13.00 and 14.00 , frozen in liquid nitrogen, and stored at $-80^{\circ} \mathrm{C}$ until extracted.

Heat or light/dark treatments were given to seedlings grown in growth chambers under cool fluorescent light ( $14 \mathrm{~h}$ light $/ 10 \mathrm{~h}$ dark), at $22^{\circ} \mathrm{C}$. Plants were used when they had two sets of true leaves. Dark treatments were administered by maintaining light-grown plants in a darkened growth chamber for 3 days. For heat shock treatments plants were moved from the illuminated chamber into a $42{ }^{\circ} \mathrm{C}$ incubator for 1 hour. RNA samples were isolated as described [5]. Total RNA was separated from DNA by precipitation with $2 \mathrm{M} \mathrm{LiCl}$. All RNA samples were separated on formaldehyde gels, transferred onto nitrocellulose filters and hybridized according to Maniatis et al. [22] using the ubiquitin or ubi3-specific probes employed for genomic clone isolation. Negatives of the ethidium-stained RNA gels and autoradiographs of northern blots were scanned using an LKB ultrascan XL laser densitometer.

Primer extension was done as described [18] using the oligo 5' -CTTCGTCTGGAGGAGAG$3^{\prime}$.

\section{Immunological blotting}

Antibodies prepared against ubiquitin and the UBI3 tail and their respective pre-immune sera were provided by Judy Callis, Dept of Biochem- istry, Univ. of California, Davis [4]. Ubiquitin antibodies were also purchased from Sigma. After SDS-PAGE, proteins were transferred to nitrocellulose and the blots were autoclaved for $20 \mathrm{~min}$ in transfer buffer $(25 \mathrm{mM}$ Tris, $193 \mathrm{mM}$ glycine $\mathrm{pH}$ 8.3). Blots were blocked in TBS (10 mM Tris pH $7.5,150 \mathrm{mM} \mathrm{NaCl}$ ) containing $2 \%$ non-fat dry milk. Antibodies were diluted 1:200 in a solution of TBS, $2 \%$ milk, $0.05 \%$ NP40, and $1 \%$ Triton X-100. Blots were shaken in the antibody solution overnight at $4{ }^{\circ} \mathrm{C}$. After washing successively in TBS, a solution comprised of TBS $+1 \%$ Triton X-100+0.05\% NP40, and TBS, blots were incubated for $1 \mathrm{~h}$ at room temperature in Protein A-alkaline phosphatase (Sigma) diluted $1: 2000$ in TBS containing $2 \%$ non-fat milk. Blots were washed in TBS, TBS $+0.05 \% \mathrm{NP} 40$, and AP $9.5(100 \mathrm{mM}$ Tris pH $9.5,100 \mathrm{mM} \mathrm{NaCl}$, $5 \mathrm{mM} \mathrm{MgCl}$ ). Blots were developed in AP 9.5 containing $0.33 \mathrm{mg} / \mathrm{ml}$ Nitroblue tetrazolium and $0.17 \mathrm{mg} / \mathrm{ml}$ bromochloroindolyl phosphate-toluidine salt.

\section{Results}

\section{cDNA clone selection and identification}

Antibodies prepared against Vicia faba 14$20 \mathrm{kDa}$ photosystem I polypeptides were initially used to screen a tomato cDNA expression library in an attempt to isolate genes encoding photosystem I polypeptides. This antiserum cross-reacted with several tomato polypeptides primarily in the $14-20 \mathrm{kDa}$ range (Fig. 1). We isolated a clone containing a $685 \mathrm{bp}$ insert having an ORF of 178 residues and theoretical molecular mass of $17.7 \mathrm{kDa}$. The predicted polypeptide was very basic with an estimated pI of 9.9. A comparison of this sequence to entries in the GenBank revealed that it was related to the ubiquitin extension protein, UBI3, cloned from yeast [26], and homologues in Drosophila [20], man [21], Arabidopsis [4] and barley [12]. The UBI3 homologues from all six species are highly conserved (Fig. 2). All the peptides are predicted to contain a 76 amino acid ubiquitin polypeptide fused to a basic 


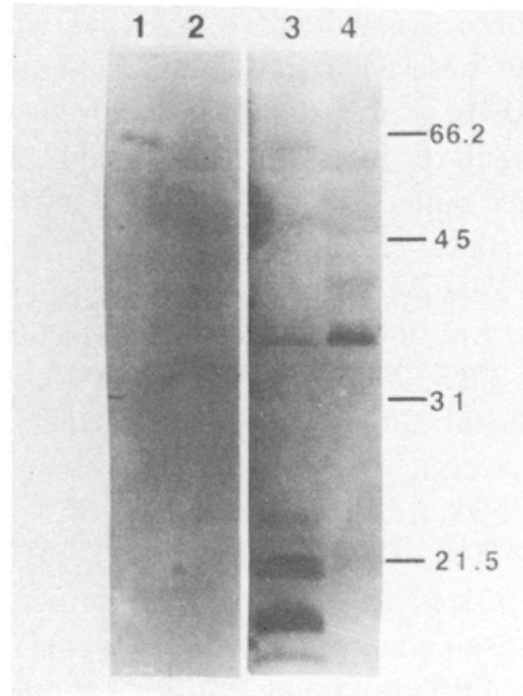

Fig. 1. Immunoblot analysis of tomato chloroplast proteins with antisera against UBI3 and PSI. Thylakoids and stroma were prepared from intact chloroplasts that were hypotonically lysed. Each lane was loaded with sample prepared from chloroplasts containing $20 \mu \mathrm{g}$ of chlorophyll. Lanes 1, 3: thylakoids; lanes 2, 4: stroma. Lanes 1 and 2 were probed with the antibody specific for UBI3. Lanes 3 and 4 were probed with antisera against PSI. Molecular weight markers (BioRad), in $\mathrm{kDa}$, are indicated to the right of the figure.

$\mathrm{C}$ terminal extension. This extension is predicted to be 80 residues for tomato, Drosophila, and humans but is 76 residues in yeast, 79 residues in barley, and 81 residues in Arabidopsis. The pre- dicted ubiquitin amino acid sequence is identical between tomato, barley, and Arabidopsis and is over $96 \%$ identical between the plant and nonplant species. The extension portion is $65-75 \%$ identical between the non-plant species but is $88 \%$ identical between tomato and Arabidopsis and over $92 \%$ identical between tomato and barley (Fig. 2). Yeast and Drosophila ubi3 genes encode an in-frame stop codon 21 and 15 bp upstream of the initiating methionine. In contrast, the tomato cDNA contained at least $66 \mathrm{bp}$ of open reading frame (Fig. 3A). Since we had isolated the tomato-ubiquitin-cDNA clone with an antibody prepared against chloroplast proteins, we tested the hypothesis that the upstream open reading frame of the tomato clone encodes a transit peptide that directs the ubiquitin polyprotein to the chloroplast and that we had isolated an incomplete cDNA. Attempts to isolate a cDNA clone containing additional $5^{\prime}$ sequence were unsuccessful, however.

\section{Isolation of ubi3 genomic clone}

To further pursue whether the upstream open reading frame encoded a genuine transit peptide, we used the cDNA clone to isolate a tomato ubi3 genomic clone. From approximately $2.5 \times 10^{5}$

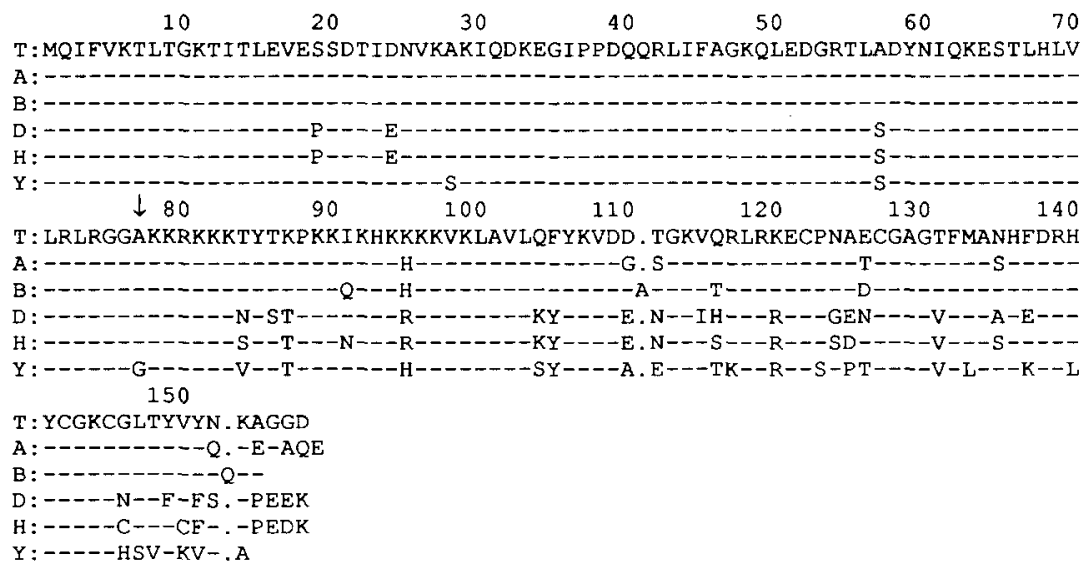

Fig. 2. Comparison of the predicted protein sequences of UBI3 and homologues. T, tomato; A, Arabidopsis (ubq6) [4]; B, barley $(m u b l)$ [12]; D, Drosophila [20]; H, human [21]; Y, yeast [26]. The first amino acid of the extension polypeptide is marked with an arrow. 


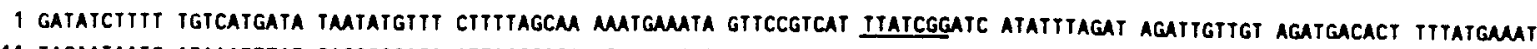
111 TACAMTAATC ATAAATTTAT CACGGGCACA AITAGCGACA ATGMGACCA ACCMUGTTA GGGGGCMUA ACCGACAGG CCAACCMMG TTATGGGTGT GCAMUMTC 221 GAATCGATCG ATAAATCGA TCGAMMUT GITATTGGG TATTTATGTT TITTCGTGGG TITATMMM MMATTATTGA ATTATTGGTT CGGTITCMT TITIATIATT

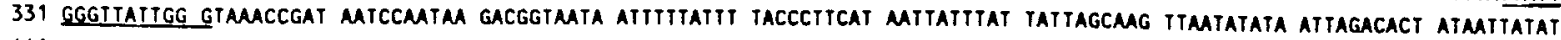

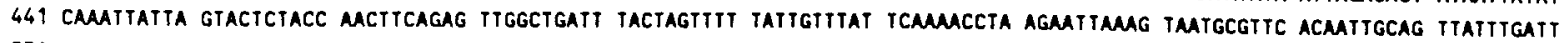

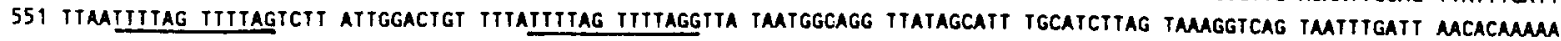

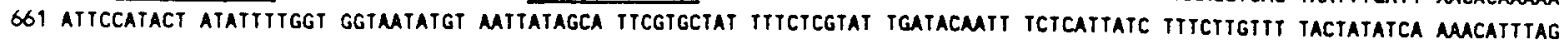

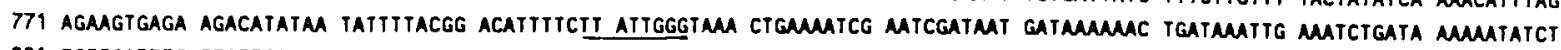

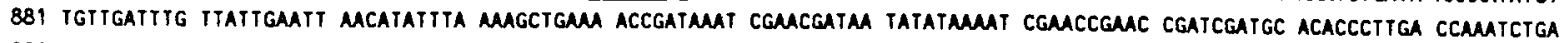

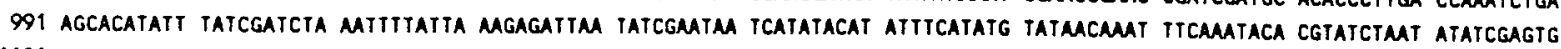

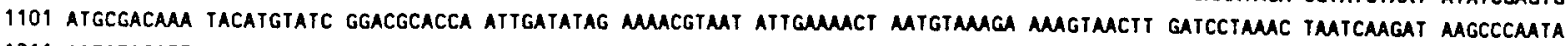

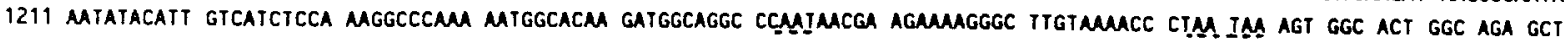

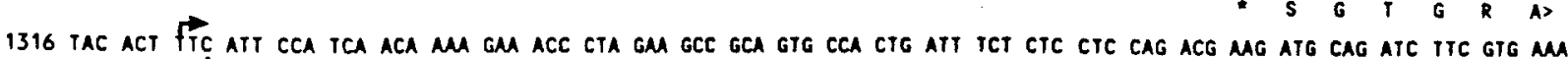

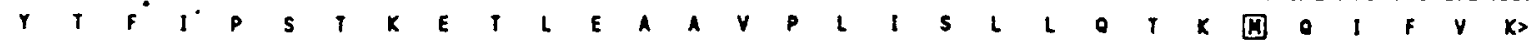
1406 ACC CTA ACG GGG MG ACG ATC ACC CTA GAG GIT GAG TCT ICC GAC ACC ATC GAC MT GTG MA GCC MG ATC CAG GAC MGG GM GGE ATT T $L$

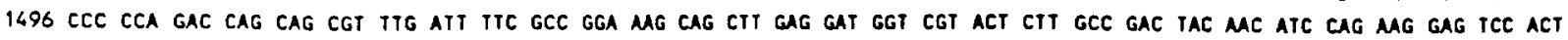

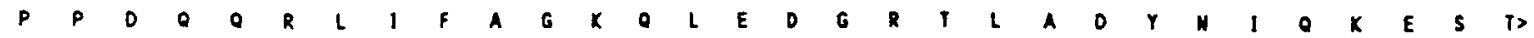
1586 CIC CAT CTC GIG CIC CGT CTC CGT GGT GGT GCT MG MG AGG MG MG MG ACC TAC ACC MG CCA MG MaG ATC MG CAC MG MG MGG

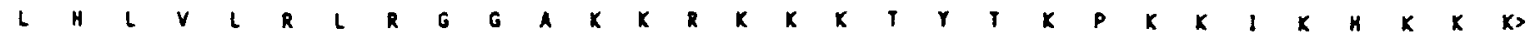
1676 MAG GTT MAG CTC GCT GTG TIG CAG ITC TAT MAG GIT GAT GAC ACT GGA MG GTT CAG AGG CTI CGT MG GAG TGC CCT MAT GCT GAG TGC

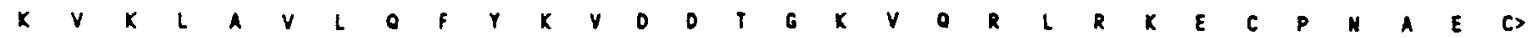
1766 GGT GCT GGA ACT TIT ATG GCT MAC CAT TIT GAC CGT CAC TAC TGT GGT MG TGT GGG CTC ACC TAC GIT TAC MC MG GCT GGA GGC GAT $G$ G G I $F$ M A N $H$ F

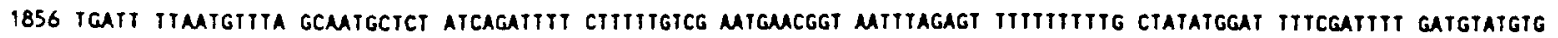
1961 ACAACCCTTG GGATTGTIGA TITATTTCM MCTMGAGT TITTGCTITA ATGTTCTCGT CTATTITCG TATCMTCTT AGTTTTATCI CATTCTAGTT GTCTMAGTI

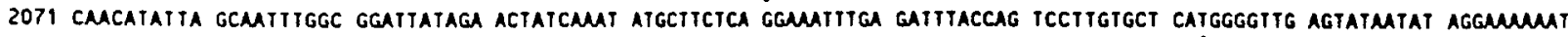
2181 AGTMATITA AGCCTGTGCT ATGTTTCTAT ACTITTATIT AITHGTCTCT GTACTTCCTC ATGCTGUUC TCTGCTGTGC ATITCATTM TITGAGMAC ATMMTMUG

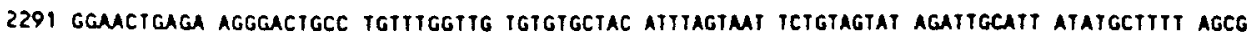

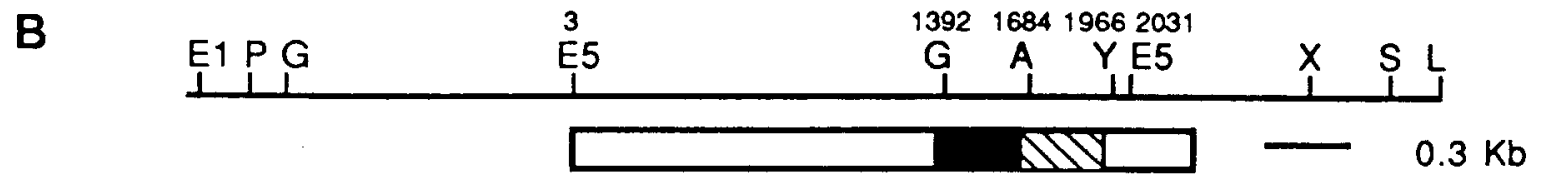

Fig. 3. DNA sequence and map of the tomato $u b i 3$ genomic clone. A. The sequence is numbered from the $5^{\prime} E c o$ RV site. The ubi3 cDNA clone begins at residue 1324 and extends to residue 2011; these nucleotides are marked with a dot. The predicted protein sequence is shown extending to the first stop codon $5^{\prime}$ to the initiating methionine (boxed). The major transcription initiation site identified by primer extension is indicated with a bent arrow. Putative TATA and CAAT boxes are underscored with dashed lines. The sequence complementary to the oligo used for primer extension begins at residue 1371 and extends to residue 1387. Direct repeats and the repetitive element TTATTGGG are underlined. B. Restriction map of the Eco RI-Sal I genomic fragment containing the $u b i 3$ gene. The boxed region was sequenced. The darkened area represents the ubiquitin sequence and the hatched area represents the UBI3 tail. Restriction sites are indicated as follows: E1, Eco RI; P, Pst; G, Bgl II; E5, Eco RV;

A, Alu I; Y, Sty I; X, Xba; S, Sac I; L, Sal I. Numbers refer to the cleavage site corresponding to the sequence in A.

phage plaques we isolated six putative ubiquitin genomic clones; only one hybridized very strongly to the $u b i 3$-specific probe (the Alu I-Sty I subfragment, see Fig. 3b). The other clones are likely to include other members of the ubiquitin gene family [2]. The cDNA probe hybridized to a single $5 \mathrm{~kb} S a l$ I-Eco RI fragment prepared from the strongly hybridizing genomic clone. This subfrag- ment was subcloned into $\mathrm{pBLUESCRIPT}$ for restriction enzyme mapping and DNA sequence analysis. The restriction map is shown in Fig. 3B. The nucleotide sequence of the $u b i 3$ gene and flanking regions are shown in Fig. 3A. The genomic clone is confirmed to encode the cDNA since the two sequences are identical within the corresponding regions (Fig. 3A). The upstream 
of the coding region, was used as a probe and it hybridized to a single fragment in the tomato genome. The F2 segregation analysis (data not shown) indicated that this fragment maps to the end of chromosome 1,23 cM from marker TG301 (S.D. Tanksley, unpublished).

\section{Expression of ubi3}

To observe whether ubi3 expression was suggestive of a role for UBI3 other than in protein synthesis, the expression characteristics of $u b i 3$ were examined using northern hybridization analysis of RNA samples extracted from various plant organs as well as young tomato leaves subjected to heat or light/dark treatments (see Materials and methods and legend for details). The cDNA hybridization probes utilized were from DNA sequences specific to the ubiquitin-coding region, to the nucleotides encoding the $u b i 3$ tail, or to the small subunit of ribulose bisphosphate carboxylase, $r b c S$. The resulting autoradiograms are shown in Figs. 5 and 6. The general ubiquitin- specific probe hybridized to transcripts of 1600 and $800 \mathrm{nt}$. The $1600 \mathrm{nt}$ species hybridized several-fold more strongly than did the $800 \mathrm{nt}$ species. The $u b i 3$-specific probe hybridized to a single $800 \mathrm{nt}$ species. Both ubiquitin transcripts were several-fold less abundant than the $r b c S$ transcript.

Analysis of total RNA prepared from plants given light and dark treatments suggests that the steady-state levels of $u b i 3 \mathrm{mRNA}$ is regulated by light. The $u b i 3$ transcript present in light-grown tomato plants was reduced $75 \%$ in light-grown plants after a 3-day continuous dark treatment (Fig. 5, lane 3A vs. 4A). The light activation observed for this gene might be modulated by the putative light regulatory elements found upstream in the genomic DNA sequence discussed in the previous section. A one-hour $42{ }^{\circ} \mathrm{C}$ heat shock also reduces the steady-state ubi3 level (Fig. 5, lane $1 \mathrm{~A}$ vs. $2 \mathrm{~A})$. In contrast, the level of the $1600 \mathrm{nt}$ species was slightly increased by dark treatment (Fig. 5, lane 3B vs. 4B) but also decreased in response to $42^{\circ} \mathrm{C}$ heat shock treatment (Fig. 5, lane 1B vs. 2B). The same blot used

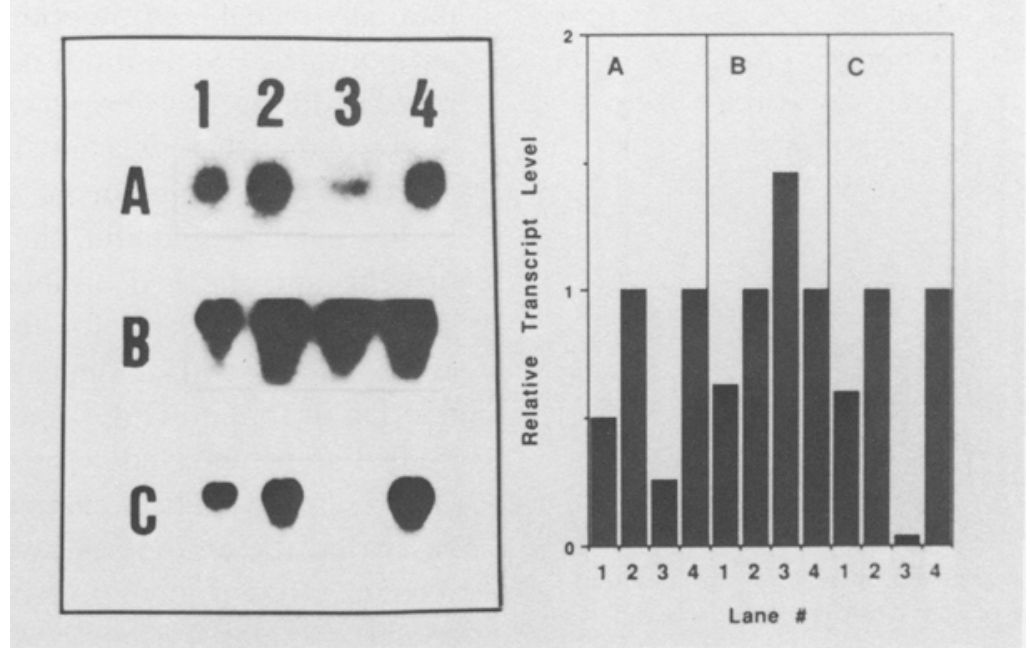

Fig. 5. RNA blot analysis of $u b i 3$ transcript levels in response to light/dark and temperature treatments. Each lane contains $8 \mu \mathrm{g}$ of total RNA prepared from tomato plants grown in the light and subjected to 1 hour of $42{ }^{\circ} \mathrm{C}$ (lane 1 ), $1 \mathrm{~h}$ of room temperature (lane 2), 3 days of darkness (lane 3), or 3 additional days of light. A. The blot was hybridized with the ubi3-specific probe and exposed against film for 7 days (only the $800 \mathrm{nt}$ area is shown). B. The same blot in A was stripped of probe, hybridized to the general ubiquitin probe, and exposed for 4 days (only the $1600 \mathrm{nt}$ area is shown). C. The same blot in B was stripped of probe and hybridized to the $r b c S$ probe and exposed for 1 day. Relative transcript level is the ratio of the hybridization signal to the signal of the ethidium-stained 16 S RNA band. The relative transcript level of the control sample in lane 2 was arbitrarily set at 1 . 
in Fig. 5A and 5B was hybridized to the $r b c S$ probe for comparison (Fig. 5C). Dark treatment resulted in barely detectable levels of $r b c S$ transcript and heat shock also reduced the steadystate level.

The steady-state level of $u b i 3$ transcript clearly exhibits an organ-specific pattern. This RNA species was most abundant in young leaves and green immature fruits (Fig. 6A, lanes 11 and 4, respectively). The transcript was also highly expressed in samples from other stages of fruit development, stems, ovaries, pedicels, and green sepals (Fig. 6A, lanes 1, 2, 3, 5, 8, 10 and 12, respectively). It was lowest in the yellow flower parts and mature leaves and petioles (Fig. 6A, lanes 6,

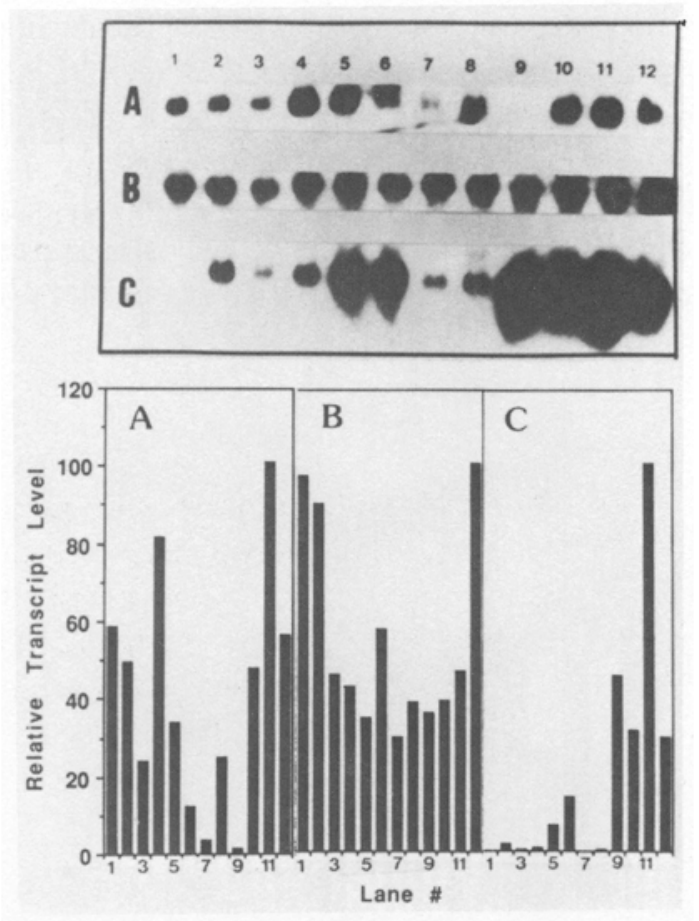

Fig. 6. Organ-specific expression of the $u b i 3$ gene in tomato. Each lane contains $8 \mu \mathrm{g}$ of total RNA prepared from different organs of field-grown tomato plants. A, B, and C as in Fig. 5 . Ripe fruit (stage 4), lane 1; mature green fruit (stage 2), lane 2; ripening fruit (stage 3), lane 3; immature green fruit (stage 1), lane 4; stems, lane 5; petioles from 4th leaves, lane 6; yellow flower petals, lane 7 ; yellow flower petals and ovaries, lane 8 ; 4 th mature leaves, lane 9; pedicels, lane 10; young 1st leaves, lane 11; and green sepals, lane 12. Relative transcript level is defined in Fig. 5. The relative transcript level was arbitrarily set to 100 for the highest expressing sample.
7 and 9). In contrast, the $1600 \mathrm{nt}$ transcript, hybridizing to the general ubiquitin probe, was strongly and uniformly expressed in every organ tissue examined (Fig. 6B, lanes 1-12). For comparison, the blot was also hybridized to tomato $r b c S$. $R b c S$ transcripts were abundant in vegetative photosynthetic tissue (Fig. 6C, lanes 5, 6, 912) including mature leaves that lacked appreciable amounts of the ubi3 transcript (Fig. 6C, lane 9). Young green fruits and ovaries contained lesser amounts of $r b c S$ transcript (Fig. 6C, lanes 2, 4 and 8) and the level was low to undetectable in flowers and ripe fruit (Fig. 6C, lanes 7, 3 and 1).

\section{Immunoblot analysis of chloroplast proteins}

Since tomato $u b i 3$ lacked a transit peptide and was nearly identical to other proteins shown to be cytoplasmically located, there was no reason to believe that UBI3 was imported into chloroplasts. We explored the possibility that UBI3 shares structural similarity to another chloroplast protein. Conceivably, if a chloroplast protein were mutually recognized by both the PSI and UBI3 antibody, the PSI antibody might recognize UBI3 protein during the library screening. We examined whether there was any immunological cross reactivity between chloroplast proteins and antibodies against ubiquitin, the ubiquitin extension protein, and the PSI antibody from Vicia faba. The antibody specific for the UBI3 tail weakly cross-reacted with a single thylakoid protein of $65 \mathrm{kDa}$ in tomato (Fig. 1, lane 1). The PSI antibody barely detected a protein of similar size (Fig. 1, lane 3). The weakness of the signal suggests that the antibodies against PSI and UBI3 recognize few if any common epitopes on chloroplast proteins. PSI antibody also did not detect up to $20 \mu \mathrm{g}$ purified ubiquitin (data not shown).

Antibodies prepared against ubiquitin (supplied by Judy Callis) reacted with two bands in a bovine ubiquitin (Sigma) sample; the lower band corresponds to free ubiquitin (Fig. 7, lane 3). The pre-immune serum (also supplied by Judy Callis) did not react with the bovine protein. Free ubiq- 


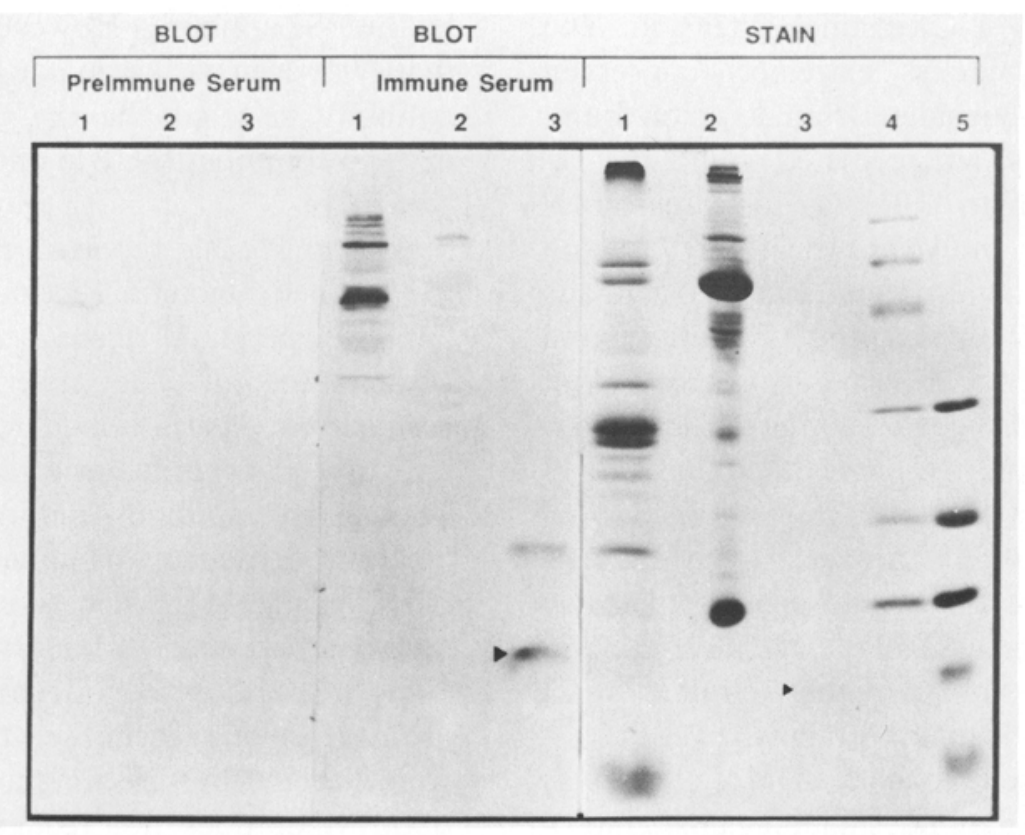

Fig. 7. Immunoblot analysis of spinach chloroplast proteins using ubiquitin antisera. Chloroplasts were treated with thermolysin as described [27]. Chloroplasts were re-isolated by spinning through $40 \%$ Percoll and washed in $0.33 \mathrm{M}$ sorbitol, $50 \mathrm{mM}$ Hepes-KOH pH 8.0. Samples were then fractionated into thylakoids (lane 1) and stroma (lane 2) after hypotonic lysis. Each lane represents fractions prepared from chloroplasts containing $20 \mu \mathrm{g}$ chlorophyll and run on $15 \%$ SDS-PAGE. Lane 3 contains $1 \mu \mathrm{g}$ bovine ubiquitin (Sigma). Lane 4: BioRad MW markers (97.4 kDa, 66.2 kDa, $45 \mathrm{kDa}, 31 \mathrm{kDa}, 21.5 \mathrm{kDa}, 14.4 \mathrm{kDa})$. Lane 5: Enprotech MW markers $(29 \mathrm{kDa}, 20.4 \mathrm{kDa}, 14 \mathrm{kDa}, 6.1 \mathrm{kDa}, 3.5 \mathrm{kDa})$. One set of samples were stained with Coomassie blue while parallel samples were transferred to nitrocellulose and probed with pre-immune serum or ubiquitin antiserum. The band corresponding to free ubiquitin is marked with a solid triangle.

uitin was not detected by the immune serum in protease-treated chloroplast samples from spinach. In a parallel experiment, the protease treatment was effective in completely degrading wheat germ translation products bound to the surface of chloroplasts (data not shown). Based on the sensitivity of the antibody to purified ubiquitin, we estimate that we would have detected ubiquitin in samples containing $1 \mathrm{ng} / \mu \mathrm{g}$ chlorophyll. The immune serum, however, reacted with higher molecular weight polypeptides found in both the thylakoid membranes and stroma (Fig. 7, lanes 1 and 2, respectively); most of the cross-reacting proteins were found in the thylakoids. The immunoreaction appears to be specific because it is unrelated to protein abundance (Fig. 7, compare immunoblot to stained gel). The immunoreaction was also observed with ubiquitin antibodies purchased from Sigma (data not shown). Further- more, pre-immune serum showed little or no binding activity toward the polypeptides detected by the immune serum. The ability of membrane and soluble polypeptides to bind ubiquitin antibodies was also observed in protease-treated pea chloroplasts (data not shown).

\section{Discussion}

Ubiquitin is encoded by at least four distinct genes in yeast all of which encode polyproteins [26]. In the present paper we report the sequence and expression characteristics of a plant homologue to the yeast gene $u b i 3$. Plant homologues to all the yeast ubiquitin genes have recently been reported $[2,3,4,12,13]$. The tomato ubi3 gene, like homologues in yeast, Arabidopsis, barley, Drosophila, and man, encodes a polyprotein consisting of 
ubiquitin fused to a C-terminal extension. The ubiquitin polypeptide is extremely conserved varying only by $2-3$ residues from ubiquitin found in other organisms. The predicted amino acid sequence of the tomato UBI3 tail most resembles that of barley and Arabidopsis (nearly $90 \%$ amino acid identity) but is also very related to humans ( $74 \%$ identity), Drosophila (69\% identity), and yeast $(65 \%$ identity). Given the high degree of conservation for this gene among the four organisms, it is reasonable to assume that tomato UBI3 is a ribosomal protein as was shown for yeast [9] and Arabidopsis [4]. In this regard it is interesting to note that the tomato $u b i 3$ promoter has five putative UAS elements and the overall consensus sequence is very similar to that found in yeast genes encoding ribosomal proteins.

Although the tomato ubi3 cDNA clone was isolated using an antibody made against chloroplast proteins, all evidence suggests that the clone does not encode a chloroplast protein. Although the cDNA does not have a stop codon upstream of the first methionine, analysis of the corresponding genomic clone revealed that an in-frame stop codon occurs downstream of a potential initiating methionine. Primer extension studies also indicate that transcription initiation occurs downstream of any other potential translation initiation sites. These data indicate that the isolated clone does not encode a form of UBI3 having a transit peptide. The predicted protein is nearly identical to an Arabidopsis homologue shown to be localized in cytoplasmic ribosomes [4] and hence it is unlikely that UBI3 is targeted to the chloroplast.

Was the $u b i 3$ clone picked inadvertently? This possibility is diminished by the fact that the clone was only detected at a frequency of less than $10^{-5}$. We attempted to address this question by examining whether chloroplasts contain proteins structurally related to ubiquitin or the UBI3 tail. We found only one tomato chloroplast protein that was recognized by UBI3-specific antibody. The detected band is much larger than UBI3 and the cross-reactivity weak, indicating that the detected protein is structurally very different than UBI3. A similarly sized protein was also detected by the PSI antibody. However, the immunoreactivity was even weaker making it unlikely that PSI antibody selected the ubi3 clone through an epitope common to UBI3 and a chloroplast protein.

In a previously reported case, St. John et al. [33] cloned ubiquitin genes using a monoclonal antibody prepared against the lymphocyte cell surface receptor. They determined that the cell surface receptor is bound to ubiquitin and the antibody was specific for an epitope on ubiquitin. Though the antibody did not recognize other ubiquitin conjugates or undenatured ubiquitin it did recognize SDS-denatured ubiquitin. In the case reported here, PSI antibodies did not detect $20 \mu \mathrm{g}$ of purified SDS-denatured ubiquitin. We are aware of one other case where ubiquitin clones were selected by screening a library with an antibody that does not recognize purified SDSdenatured ubiquitin (Kirk Apt, personal communication). In this example, polyubiquitin genes from the red alga, Callithamnion neglectum were isolated by probing with an antibody made against purified phycobilisome proteins. One possibility we did not explore is whether the $E$. coli-expressed ubiquitin has a strong affinity for the antibody or one of the screening reagents. This affinity would need to be a property of the native or recombinant protein because SDS-denatured purified ubiquitin was not detected by the PSI or phycobilisome antibodies on western blots. PSI antibodies also did not detect SDS-denatured fusion protein.

Ubiquitin has not been found in mitochondria or bacteria [15]. Wettern et al. [36] recently reported evidence that ubiquitin or ubiquitin conjugates are found in Chlamydomonas chloroplasts. Furthermore, they speculated that ubiquitin might enter the chloroplast in the form of protein conjugates. At the time of this writing, they had not extended their observations to higher-plant chloroplasts (M. Wettern, personal communication). We were unable to detect any free ubiquitin with ubiquitin antibodies in chloroplasts from spinach or pea. However, in both organisms we did find higher-molecular-weight forms that reacted with the ubiquitin antibody, consistent with the observations of Wettern et al. [36]. The fact that pro- 
tease treatment of intact chloroplasts does not diminish the signal indicates that the affected proteins are within the chloroplast. In lieu of the apparent absence of any chloroplast routing signals on ubiquitin proteins, the simplest explanation is that nuclear-encoded chloroplast proteins are ubiquitinated in the cytosol and are still capable of being imported into the chloroplast.

Further biochemical studies are required to establish whether the proteins detected by the antibody are indeed ubiquitinated and are not fortuitously cross-reacting with the antibody. If the proteins are ubiquitinated, it will be interesting to identify whether they are all nuclear-encoded. If chloroplast-encoded proteins are also ubiquitinated, it would be consistent with the recent report that lysed chloroplasts are capable of ubiquitinating chloroplast polypeptides [35]. The authors of this report speculated that a ubiquitin conjugating system may be involved in regulating protein turnover in chloroplasts. Conceivably, the ubiquitin for this process could be salvaged from imported conjugates. It will also be interesting to observe whether ubiquitination has an effect on the import of proteins into the chloroplast. In this context it is interesting to note the report that ubiquitination promotes the translocation of monoamine oxidase $B$ into the mitochondrial outer membrane [37].

Other investigators have observed four size classes of ubiquitin transcripts expressed in most tissues of Arabidopsis and barley [2, 4, 11]. Probes specific for the extension protein genes only hybridize to the smallest class between $0.75-1.00 \mathrm{~kb}$ in Arabidopsis [4]. In tomato we observed only two size classes, of 1.6 and $0.8 \mathrm{~kb}$ transcripts, hybridizing to a general ubiquitin probe. We also found that a $u b i 3$ specific probe only hybridized to the $0.8 \mathrm{~kb}$ transcript. The larger transcript might be encoded by a homologue to the polyubiquitin gene, $u b q 4$ [2]. Ubq4 encodes a $1.35 \mathrm{~kb}$ transcript in Arabidopsis [2]. Both size classes of tomato ubiquitin transcript were down-regulated by a $42{ }^{\circ} \mathrm{C}$ heat shock. Similarly, Burke et al. [2] previously found that 0.7 and $1.35 \mathrm{~kb}$ ubiquitin transcripts were down-regulated by heat shock in Arabidopsis. We did not observe, as they had found, that a $1.7 \mathrm{~kb}$ ubiquitin transcript was upregulated by heat shock. In yeast, the $u b i 4$ gene is up-regulated and $u b i 3$ continues to be expressed during heat shock while $u b i l$ and $u b i 2$ are downregulated [10].

The tomato $u b i 3$ transcript is most abundant in light-grown and young tissues consistent with its presumed role in protein synthesis. We found that it was nearly absent in flower petals, mature lightgrown leaves, and young light-grown leaves kept in darkness for three days. In contrast, the $1600 \mathrm{~kb}$ ubiquitin transcript showed high levels of expression in all samples. The different patterns of expression between the $u b i 3$ transcript and the $1600 \mathrm{~kb}$ ubiquitin transcript is further evidence for different functions for the proteins these transcripts encode, a ribosomal protein in one case and presumably a component of the proteolytic system in the other. Callis et al. [4] similarly found that levels of expression for the extension protein genes were lower in mature leaf tissue. Although they did not examine transcript levels in darkadapted plants, they immunologically detected extension protein in dark-grown 3-day-old seedlings. Conceivably the expression of the extension protein in the dark-grown seedlings is developmentally programmed to coincide with a requirement for active protein synthesis during germination and seedling emergence whereas in slightly older photosynthetic tissue dark adaptation is a cue to curtail protein synthesis and hence to reduce expression of the extension protein.

\section{Acknowledgements}

C.I.W.-D.P.B. Publication No. 1107. Support for this work was provided by National Institutes of Health grant GM42609-02 to N.E.H. and a grant from the Natural Sciences and Engineering Research Council to K.K. We thank Zdenko Ko for her expert technical assistance, Judy Callis for providing ubiquitin antiserum and valuable discussion, and W. Gruissem for the tomato genomic library. 


\section{References}

1. Bernatzky R, Tanksley SD: Toward a saturated linkage map in tomato based on isozymes and random cDNA sequences. Genetics 112: 887-898 (1986).

2. Burke TJ, Callis J, Vierstra RD: Characterization of a polyubiquitin gene from Arabidopsis thaliana. Mol Gen Genet 213: 435-443 (1988).

3. Callis J, Pollmann L, Shanklin J, Wettern M, Vierstra RD: Sequence of a cDNA from Chlamydomonas reinhardii encoding a ubiquitin-52 amino acid extension protein. Nucl Acids Res 17: 8377 (1989).

4. Callis J, Raasch JA, Vierstra RD: Ubiquitin extension proteins of Arabidopsis thaliana - Structure, localization, and expression of their promoters in transgenic tobacco. J Biol Chem 265: 12486-12493 (1990).

5. Cashmore AR: The isolation of poly $\mathrm{A}^{+}$messenger RNA from higher plants. In: Edelman M, Hallick RB, Chua $\mathrm{N}-\mathrm{H}$ (eds) Methods in Chloroplast Molecular Biology, pp. 387-392. Elsevier Biomedical Press, Amsterdam (1982).

6. Chau V, Tobias JW, Bachmair A, Marriott D, Ecker DJ, Gonda DK, Varshavsky A: A multiubiquitin chain is confined to specific lysine in a targeted short-lived protein. Science 243: 1576-1583 (1989).

7. Cheng MY, Hartl F-U, Martin J, Pollack RA, Kalousek F, Neupert W, Hallberg E, Hallberg R, Horwich AL: Mitochondrial heat-shock protein hsp60 is essential for assembly of proteins imported into yeast mitochondria. Nature 337: 620-625 (1989).

8. Datta N, Cashmore AR: Binding of a pea nuclear protein to promoters of certain photoregulated genes is modulated by phosphorylation. Plant Cell 1: 1069-1077 (1989).

9. Finley D, Bartel B, Varshavsky A: The tails of ubiquitin precursors are ribosomal proteins whose fusion to ubiquitin facilitates ribosome biogenesis. Nature 338: 394-401 (1989).

10. Finley D, Ozkaynak E, Jentsch S, McGrath JP, Bartel B, Pazin M, Snapka RM, Varshavsky A: Molecular genetics of the ubiquitin system. In: Rechsteiner M (ed) Ubiquitin, pp. 39-75. Plenum Press, New York/London (1988).

11. Gausing K, Barkardottir R: Structure and expression of ubiquitin genes in higher plants. Eur J Biochem 158: 5762 (1986)

12. Gausing $\mathrm{K}$, Jensen $\mathrm{CB}: 2$ Ubiquitin long-tail fusion genes arranged as closely spaced direct repeats in barley. Gene 94: 165-171 (1990).

13. Genschik P, Parmentier Y, Criqui MC, Fleck J: Sequence of a ubiquitin carboxyl extension protein of Nicotiana tabacum. Nucl Acids Res 18: 4007 (1990).

14. Giuliano G, Pichersky E, Malik VS, Timko MP, Scolnick PA, Cashmore AR: An evolutionarily conserved protein binding sequence upstream of a plant light-regulated gene. Proc Natl Acad Sci USA 85: 7089-7093 (1988).
15. Goff SA, Voellmy R, Goldberg AL: Protein breakdown and the heat shock response. In: Rechsteiner M (eds) Ubiquitin, pp. 207-238. Plenum Press, New York/ London (1988).

16. Green PJ, Yong M-H, Cuozzo M, Kano-Murakami Y, Silverstein $\mathrm{P}$, Chua $\mathrm{N}-\mathrm{H}$ : Binding site requirements for pea nuclear protein factor GT-1 correlate with sequences required for light-dependent transcriptional activation of the $r b c S-3 A$ gene. EMBO J 7: 4035-4044 (1988).

17. Hoffman NE, Pichersky E, Malik VS, Castresana C, Ko $\mathrm{K}$, Darr SC, Cashmore AR: A cDNA clone encoding a photosystem I protein with homology to photosystem II chlorophyll $a / b$-binding polypeptides. Proc Natl Acad Sci USA 84: 8844-8848 (1987).

18. Kellmann JW, Pichersky E, Piechulla B: Analysis of the diurnal expression patterns of the tomato chlorophyll $a$ / $b$-binding protein genes - Influence of light and characterization of the gene family. Photochem Photobiol 52: 35-41 (1990).

19. Kuhlemeier C, Cuozzo M, Green PJ, Goyvaerts E, Ward $\mathrm{K}$, Chua N-H: Localization and conditional redundancy of regulatory elements in $r b c S-3 A$, a pea gene encoding the small subunit of ribulose-bisphosphate carboxylase. Proc Natl Acad Sci USA 85: 4662-4666 (1988).

20. Lee H, Simon J, Lis JT: Structure and expression of ubiquitin genes of Drosophila melanogaster. Mol Cell Biol 8: 4727-4735 (1988).

21. Lund PK, Moats-Staats BM, Simmons JG, Hoyt E, D'Ercole AJ, Martin F, Van Wyk JJ: Nucleotide sequence analysis of a cDNA encoding human ubiquitin reveals that ubiquitin is synthesized as a precursor. $\mathbf{J}$ Biol Chem 260: 7609-7613 (1985).

22. Maniatis T, Fritsch EF, Sambrook J: Molecular Cloning: A Laboratory Manual. Cold Spring Harbor Laboratory, Cold Spring Harbor, NY (1982).

23. Mullertaubenberger A, Graack HR, Grohmann L, Schleicher M, Gerisch G: An extended ubiquitin of Dictyostelium is located in the small ribosomal subunit. J Biol Chem 264: 5319-5322 (1989).

24. Mullet JE, Burk JJ, Arntzen CJ: Chlorophyll proteins of Photosystem I. Plant Physiol 65: 814-822 (1980).

25. Ostermann J, Horwich AL, Neupert W, Hartl FU: Protein folding in mitochondria requires complex formation with Hsp60 and ATP hydrolysis. Nature 341: 125-130 (1989).

26. Ozkaynak E, Finley D, Solomon MJ, Varshavsky A: The yeast ubiquitin genes: a family of natural gene fusions. EMBO J 6: 1429-1439 (1987).

27. Pichersky E, Hoffman NE, Malik VS, Bernatzky R, Tanksley SD, Szabo L, Cashmore AR: The tomato cab-4 and $c a b-5$ genes encode a second type of CAB polypeptides localized in photosystem II. Plant Mol Biol 9: 109. 120 (1987).

28. Planta RJ, Raue HA: Control of ribosome biogenesis in yeast. Trends Genet 4: 64-68 (1988).

29. Reading DS, Hallberg RL, Myers AM: Characterization 
of the yeast HSP60 gene coding for a mitochondrial assembly factor. Nature 337: 655-659 (1989).

30. Redman KL, Rechsteiner M: Identification of the long ubiquitin extension as ribosomal protein $\mathrm{S} 27 \mathrm{a}$. Nature 338: 438-440 (1989).

31. Sanger F, Nicklen $S$, Coulson AR: DNA sequencing with chain terminating inhibitors. Proc Natl Acad Sci USA 74: 5463-5467 (1977).

32. Shanklin J, Jabben M, Vierstra RD: Red light formation of ubiquitin-phytochrome conjugates: identification of possible intermediates of phytochrome degradation. Proc Natl Acad Sci USA 84: 359-363 (1987).

33. St. John T, Gallatin M, Siegelman M, Smith HT, Fried VA, Weissman IL: Expression cloning of a lymphocyte homing receptor CDNA: Ubiquitin is the reactive species. Science 231: 845-850 (1986).
34. Sugita M, Manzara T, Pichersky E, Cashmore A, Gruissem W: The genomic organization, sequence analysis, and expression of all five genes encoding the small subunit of ribulose 1,5-bisphosphate carboxylase from tomato. Mol Gen Genet 209: 247-256 (1987).

35. Veierskov B, Ferguson IB: Conjugation of ubiquitin to proteins from green plant tissues. Plant Physiol 96: 4-9 (1991).

36. Wettern M, Parag HA, Pollmann L, Ohad I, Kulka RG: Ubiquitin in Chlamydomonas reinhardtii - Distribution in the cell and effect of heat shock and photoinhibition on its conjugate pattern. Eur J Biochem 191: 571-576(1990).

37. Zhaung $\mathrm{Z}$, McCauley $\mathbf{R}$ : Ubiquitin is involved in the in vitro insertion of monamine oxidase $B$ into mitochondrial outer membranes. J Biol Chem 264: 14594-14596 (1989). 